\title{
Well-Posedness and Comparison Principle for Option Pricing with Switching Liquidity
}

\author{
T.B. Gyulov and L.G. Valkov* \\ University of Ruse "Angel Kanchev", 7017 Ruse, Bulgaria
}

June 28, 2021

\begin{abstract}
We consider an integro-differential equation derived from a system of coupled parabolic PDE and an ODE which describes an European option pricing with liquidity shocks. We study the well-posedness and prove comparison principle for the corresponding initial value problem.
\end{abstract}

\section{Introduction}

This work is devoted to the study of an initial value problem of the following form

$$
\left\{\begin{aligned}
\frac{\partial u}{\partial \tau}-\frac{1}{2} \sigma^{2} S^{2} \frac{\partial^{2} u}{\partial S^{2}} & =-\nu_{01} e^{u(S, \tau)}\left(\nu_{10} \int_{0}^{\tau} e^{-u(S, s)} d s+e^{-\gamma h(S)}\right)+\kappa \\
u(S, 0) & =\gamma h(S) .
\end{aligned}\right.
$$

Here $\tau \in[0, T], S \in(0,+\infty), h(S)$ is a given function and $\sigma, \nu_{01}, \nu_{10}, \kappa$ and $\gamma$ are constants.

The integro-differential equation in (1) is derived from a system of coupled parabolic PDE and ODE which is suggested by M. Ludkovski and Q. Shen [6] in European option pricing in a financial market switching between two states -a liquid state (0) and an illiquid (1) one. We briefly describe their model. First, it is assumed that the dynamics of the liquidity is represented by a continuous-time Markov chain $\left(M_{t}\right)$ with intensity rates of the transitions $0 \rightarrow 1$ and $1 \rightarrow 0$ and determined by the constants $\nu_{01}$ and $\nu_{10}$, respectively. During the liquid phase $\left(M_{t}=0\right)$ the market dynamics follows the classical Black-Scholes model. More precisely, the price $S_{t}$ of a stock is modelled by geometric Brownian motion

$$
d S_{t}=\mu S_{t} d t+\sigma S_{t} d W_{t},
$$

with drift $\mu$ and volatility $\sigma$ and a standard one-dimensional Brownian motion $\left(W_{t}\right)$ which is independent of the Markov chain $\left(M_{t}\right)$ (under the "real world" probability $\mathbb{P})$. Then the wealth process $\left(X_{t}\right)$ satisfies

$$
d X_{t}=\mu \pi_{t} X_{t} d t+\sigma \pi_{t} X_{t} d W_{t},
$$

*e-mails: tgulov@uni-ruse.bg (T.Gyulov) and lvalkov@uni-ruse.bg (L.Valkov) 
where $\pi_{t}$ denotes the proportion of stock holdings in the total wealth $X_{t}$. For simplicity, it is assumed that the interest rate of the riskless asset is zero.

Respectively, in the illiquid phase $\left(M_{t}=1\right)$, the market is static and trading in stock is not permitted, i.e., $d S_{t}=d X_{t}=0$.

The presence of liquidity shocks is a source of non-traded risk and makes the market incomplete. Ludkovski and Shen investigate expected utility maximization with exponential utility function:

$$
u(x)=-e^{-\gamma x},
$$

where $\gamma>0$ is the investor's risk aversion parameter. The value functions $\hat{U}^{i}(t, X, S), i=0,1$ for the optimal investment problem are defined as follows:

$$
\hat{U}^{i}(t, X, S):=\sup _{\pi_{t}} \mathbb{E}_{t, X, S, i}^{\mathbb{P}}\left[-e^{-\gamma\left(X_{T}+h\left(S_{T}\right)\right)}\right], \quad i=0,1
$$

where $\mathbb{E}_{t, X, S, i}^{\mathbb{P}}$ is the expectation under the measure $\mathbb{P}$ with starting values $S_{t}=$ $S, X_{t}=X$ and $M_{t}=i$. The supremum above is taken over all admissible trading strategies $\left(\pi_{t}\right)$ and the function $h(S)$ denotes the terminal payoff of a contingent claim. Standard stochastic control methods and the properties of the exponential utility function imply that the value functions can be presented by

$$
\hat{U}^{i}(t, X, S)=-e^{-\gamma X} e^{-\gamma R^{i}(t, S)}, \quad i=0,1,
$$

where $R^{i}(t, S)$ are the unique viscosity solutions of the system ([6])

$$
\left\{\begin{array}{l}
R_{t}^{0}+\frac{1}{2} \sigma^{2} S^{2} R_{S S}^{0}-\frac{\nu_{01}}{\gamma} e^{-\gamma\left(R^{1}-R^{0}\right)}+\frac{d_{0}+\nu_{01}}{\gamma}=0 \\
R_{t}^{1}-\frac{\nu_{10}}{\gamma} e^{-\gamma\left(R^{0}-R^{1}\right)}+\frac{\nu_{10}}{\gamma}=0
\end{array}\right.
$$

with the terminal condition $R^{i}(T, S)=h(S), i=0,1$. Here $d_{0}:=\mu^{2} / 2 \sigma^{2}$.

Let $p$ and $q$ denote the buyer's indifference prices corresponding to liquid and illiquid initial state respectively. They are defined as follows: $\hat{U}^{0}(t, X-$ $p, S)=\hat{V}^{0}(t, X)$ and $\hat{U}^{1}(t, X-q, S)=\hat{V}^{1}(t, X)$ where $\hat{V}^{i}, i=0,1$ are the value functions of the Merton optimal investment problem (i.e. the case when $h(S) \equiv 0)$. It can be shown that $p$ and $q$ satisfy a system of differential equations which is quite similar to (2) (see (15)). In fact,

$$
p=R^{0}+\gamma^{-1} \ln F_{0}(t) \quad \text { and } \quad q=R^{1}+\gamma^{-1} \ln F_{1}(t)
$$

where

$$
\begin{aligned}
F_{0}(t) & =c_{1} e^{\lambda_{1} t}+c_{2} e^{\lambda_{2} t} \\
F_{1}(t) & =\frac{1}{\nu_{01}}\left(c_{1}\left(d_{0}+\nu_{01}-\lambda_{1}\right) e^{\lambda_{1} t}+c_{2}\left(d_{0}+\nu_{01}-\lambda_{2}\right) e^{\lambda_{2} t}\right) \\
\lambda_{1,2} & =\frac{d_{0}+\nu_{01}+\nu_{10} \pm \sqrt{\left(d_{0}+\nu_{01}+\nu_{10}\right)^{2}-4 d_{0} \nu_{10}}}{2}, \\
c_{1} & =\frac{\lambda_{2}-d_{0}}{\lambda_{2}-\lambda_{1}} e^{-\lambda_{1} T}, \quad \text { and } \quad c_{2}=\frac{\lambda_{1}-d_{0}}{\lambda_{1}-\lambda_{2}} e^{-\lambda_{2} T} .
\end{aligned}
$$


Indifference pricing was first used in the pioneering paper of Hodges and Neuberger [3]. We refer also to [2] for further applications (see [4] and [8] as well).

The existence of classical solutions was proved in [6] when the payoff function $h(S)$ is bounded. This case is restrictive since it does not include such typical example as the call option $h=\max \{S-K, 0\}$ with strike price $K$. We investigate the solvability of the problem and prove the existence and uniqueness of a weak solution in suitable Sobolev weighted spaces which allows unbounded terminal payoff functions.

The integro-differential equation (11) is derived from (2) as follows. Denote $r^{0}:=\gamma R^{0}, r^{1}=\gamma R^{1}$. The system of differential equations for $r^{0}$ and $r^{1}$ has the following from:

$$
\left\{\begin{array}{l}
r_{\tau}^{0}-\frac{1}{2} \sigma^{2} S^{2} r_{S S}^{0}=-\nu_{01} e^{-\left(r^{1}-r^{0}\right)}+d_{0}+\nu_{01} \\
r_{\tau}^{1}=-\nu_{10} e^{-\left(r^{0}-r^{1}\right)}+\nu_{10}
\end{array}\right.
$$

where $\tau=T-t$. The ODE in (3) can be solved explicitly with respect to $r^{1}$. Then we obtain the initial value problem (1) under the substitution $u:=$ $r^{0}-\nu_{10} \tau$ and $\kappa:=d_{0}+\nu_{01}-\nu_{10}$.

The paper is organized as follows. In Section 2 we prove a comparison principle (Theorem 2.1) for classical solutions to the problem (11). Then, in Section 3 we prove a comparison principle (Theorem 3.4) for weak sub/super solutions. In addition, we study the existence and uniqueness of weak solutions in a suitable weighted Sobolev space (see Theorem 3.77).

\section{Comparison principle for classical solutions}

In this section we consider solutions of (1) satisfying

$$
|u|,|h| \leq A \exp \left(\alpha \ln ^{2} S\right)=A S^{\alpha \ln S},
$$

for some positive constants $A$ and $\alpha$. Note that conditions (4) include for example linear growth, polinomial and powers of $S$ with arbitrary exponent.

We prove the following comparison principle:

Theorem 2.1. Let $u_{1}, u_{0} \in C((0,+\infty) \times[0, T)) \cap C^{2,1}((0,+\infty) \times(0, T))$ be two clasical solutions of (11) corresponding to the initial data $h=h_{1}$ and $h=h_{0}$, respectively and such that the conditions (4) hold. Then

$$
\gamma \inf \left(h_{1}-h_{0}\right) \leq u_{1}-u_{0} \leq \gamma \sup \left(h_{1}-h_{0}\right)
$$

We will only prove the lower bound in (5) since the upper one follows immediately from it. In addition, we can assume that

$$
\underline{h}:=\gamma \inf \left(h_{1}-h_{0}\right)>-\infty,
$$

otherwise the left inequality in (5) is trivial. We will use the following auxiliary lemma 
Lemma 2.2. Let $u_{1}$ and $u_{0}$ be as in Theorem 2.1 and $\tau_{1} \geq 0$ be such that $u_{1}(S, \tau)-u_{0}(S, \tau) \geq \underline{h}$ for any $\tau \in\left[0, \tau_{1}\right]$. Then, there exists a constant $\bar{\tau}>0$ such that $u_{1}(S, \tau)-u_{0}(S, \tau) \geq \underline{h}$ for any $\tau \in\left[0, \tau_{1}+\bar{\tau}\right)$. In addition, $\bar{\tau}$ depends only on $\alpha$ defined in (4) and $\sigma$.

Proof. Let $u_{1}$ and $u_{0}$ be two solutions of (11) corresponding to the initial conditions $u_{1}(S, 0)=\gamma h_{1}(S)$ and $u_{0}(S, 0)=\gamma h_{0}(S)$. Denote $\tilde{u}=u_{1}-u_{0}$, $\tilde{h}=\gamma\left(h_{1}-h_{0}\right), u_{\xi}=\xi u_{1}+(1-\xi) u_{0}, h_{\xi}=\xi h_{1}+(1-\xi) h_{0}$, for $\xi \in[0,1]$ and define

$$
\mathcal{F}[\tau ; u, g]:=-\nu_{01} e^{u(\tau)}\left(\nu_{10} \int_{0}^{\tau} e^{-u(s)} d s+e^{-g}\right)+\kappa .
$$

Then

$$
\begin{gathered}
\mathcal{F}\left[\tau ; u_{1}, \gamma h_{1}\right]-\mathcal{F}\left[\tau ; u_{0}, \gamma h_{0}\right]=\int_{0}^{1} \frac{d}{d \xi}\left(\mathcal{F}\left[\tau ; u_{\xi}, \gamma h_{\xi}\right]\right) d \xi \\
=-\nu_{01} \tilde{u} \int_{0}^{1} e^{u_{\xi}(\tau)}\left(\nu_{10} \int_{0}^{\tau} e^{-u_{\xi}(s)} d s+e^{-\gamma h_{\xi}}\right) d \xi \\
\quad+\nu_{01} \int_{0}^{1} e^{u_{\xi}(\tau)}\left(\nu_{10} \int_{0}^{\tau} e^{-u_{\xi}(s)} \tilde{u}(s) d s+e^{-\gamma h_{\xi}} \tilde{h}\right) d \xi \\
=-\nu_{01} \nu_{10} \int_{0}^{1} \int_{0}^{\tau} e^{u_{\xi}(\tau)-u_{\xi}(s)}(\tilde{u}(\tau)-\tilde{u}(s)) d s d \xi \\
\quad-\nu_{01}(\tilde{u}(\tau)-\tilde{h}) \int_{0}^{1} e^{u_{\xi}(\tau)-\gamma h_{\xi}} d \xi
\end{gathered}
$$

and

$$
\begin{gathered}
\tilde{u}_{\tau}-\frac{1}{2} \sigma^{2} S^{2} \tilde{u}_{S S}=- \\
-\nu_{01} \nu_{10} \int_{0}^{\tau}(\tilde{u}(\tau)-\tilde{u}(s)) d s \int_{0}^{1} e^{u_{\xi}(\tau)-u_{\xi}(s)} d \xi \\
-\nu_{01}(\tilde{u}(\tau)-\tilde{h}) \int_{0}^{1} e^{u_{\xi}(\tau)-\gamma h_{\xi}} d \xi
\end{gathered}
$$

Next, define

$$
\omega(S, \tau):=\frac{1}{\sqrt{T_{1}-\tau}} \exp \left(\frac{\left(\ln S-\frac{1}{2} \sigma^{2}\left(T_{1}-\tau\right)\right)^{2}}{2 \sigma^{2}\left(T_{1}-\tau\right)}\right),
$$

where $T_{1}>0$ and $(S, \tau) \in(0,+\infty) \times\left[0, T_{1}\right)$. Note that $\mathcal{L}_{B S} \omega=\omega_{\tau}-$ $\frac{1}{2} \sigma^{2} S^{2} \omega_{S S}=0$ and $\omega$ is increasing with respect to $\tau$ in the interval $\tau \in$ $\left[T_{1}-4 / \sigma^{2}, T_{1}\right)$. Choose $T_{1}>\tau_{1}$ in (10) such that the inequality

$$
\alpha<\frac{1}{2 \sigma^{2}\left(T_{1}-\tau\right)},
$$

holds for all $\tau \in\left[\tau_{1}, T_{1}\right)$ and $T_{1}-4 / \sigma^{2}<\tau_{1}$. It is enough to define $T_{1}:=\tau_{1}+\bar{\tau}$, 
where $0<\bar{\tau}<\min \left\{\left(2 \sigma^{2} \alpha\right)^{-1}, 4 / \sigma^{2}\right\}$. Next, let $\varphi_{\epsilon}=\tilde{u}+\epsilon \omega$. Then

$$
\begin{aligned}
\left(\varphi_{\epsilon}\right)_{\tau} & -\frac{1}{2} \sigma^{2} S^{2}\left(\varphi_{\epsilon}\right)_{S S}=-\nu_{01} \nu_{10} \int_{0}^{\tau}(\tilde{u}(\tau)-\tilde{u}(s)) d s \int_{0}^{1} e^{u_{\xi}(\tau)-u_{\xi}(s)} d \xi \\
- & \nu_{01}(\tilde{u}(\tau)-\tilde{h}) \int_{0}^{1} e^{u_{\xi}(\tau)-\gamma h_{\xi}} d \xi \\
\geq & -\nu_{01} \nu_{10}(\tilde{u}(\tau)-\underline{h}) \int_{0}^{\tau_{1}} d s \int_{0}^{1} e^{u_{\xi}(\tau)-u_{\xi}(s)} d \xi \\
& -\nu_{01} \nu_{10} \int_{\tau_{1}}^{\tau}(\tilde{u}(\tau)-\tilde{u}(s)) d s \int_{0}^{1} e^{u_{\xi}(\tau)-u_{\xi}(s)} d \xi \\
- & \nu_{01}(\tilde{u}(\tau)-\tilde{h}) \int_{0}^{1} e^{u_{\xi}(\tau)-\gamma h_{\xi}} d \xi
\end{aligned}
$$

We will prove that $\varphi_{\epsilon} \geq \underline{h}$ for any $\tau \in\left[\tau_{1}, T_{1}\right)$. Indeed, assume by contradiction that inf $\varphi_{\epsilon}<\underline{h}$. Note that $\left.\varphi_{\epsilon}\right|_{\tau=\tau_{1}}>\underline{h}$ and there exist $\bar{S}$ and $\underline{S}$ such that $\varphi_{\epsilon}>\underline{h}$ if either $S \leq \underline{S}$ or $S \geq \bar{S}$. In fact, $\varphi_{\epsilon} \rightarrow+\infty$ uniformly when either $|\ln S| \rightarrow+\infty$ or $\tau \rightarrow T_{1}$. The last observations imply that $\varphi_{\epsilon}$ attains minimum in an interior point $\left(S_{*}, \tau_{*}\right) \in(\underline{S}, \bar{S}) \times\left(\tau_{1}, T_{1}\right)$ and $\varphi_{\epsilon}\left(S_{*}, \tau_{*}\right)<\underline{h}$. Then, $\left(\varphi_{\epsilon}\right)_{\tau}\left(S_{*}, \tau_{*}\right)=0$, $\left(\varphi_{\epsilon}\right)_{S S}\left(S_{*}, \tau_{*}\right) \geq 0$ and

$$
\begin{aligned}
\tilde{u}\left(S_{*}, \tau_{*}\right)-\tilde{h} \leq & \tilde{u}\left(S_{*}, \tau_{*}\right)-\underline{h}=\varphi_{\epsilon}\left(S_{*}, \tau_{*}\right)-\underline{h}-\epsilon \omega\left(S_{*}, \tau_{*}\right)<0 \\
\tilde{u}\left(S_{*}, \tau_{*}\right)-\tilde{u}\left(S_{*}, s\right)= & \varphi_{\epsilon}\left(S_{*}, \tau_{*}\right)-\varphi_{\epsilon}\left(S_{*}, s\right) \\
& -\epsilon\left(\omega\left(S_{*}, \tau_{*}\right)-\omega\left(S_{*}, s\right)\right)<0, \quad \forall s \in\left[\tau_{1}, \tau_{*}\right],
\end{aligned}
$$

since $\omega$ is increasing in $\tau$. Thus the right hand side of (12) is positive, a contradiction. Hence $\varphi_{\epsilon}=\tilde{u}+\epsilon \omega \geq \underline{h}$ for any $\tau \in\left[\tau_{1}, T_{1}\right)$. Let $\epsilon \rightarrow 0$. Then $\tilde{u}=u_{1}-u_{0} \geq \underline{h}$ for any $\tau \in\left[\tau_{1}, T_{1}\right)$.

Proof. (of Theorem 2.11) The comparison principle follows by induction and the auxiliary Lemma 2.2] we first take $\tau_{1}=0$ and prove it in the interval $[0,1 / 2 \bar{\tau}]$, then let $\tau_{1}=1 / 2 \bar{\tau}$ and consider the interval $[1 / 2 \bar{\tau}, \bar{\tau}]$ and etc.

Now, as a corollary we formulate comparison principle for the buyer's indifference prices $p(S, t), q(S, t)$ which satisfy the terminal value problem

$$
\left\{\begin{array}{l}
p_{t}+\frac{1}{2} \sigma^{2} S^{2} p_{S S}-\frac{\nu_{01}}{\gamma} \frac{F_{1}}{F_{0}} e^{-\gamma(q-p)}+\frac{d_{0}+\nu_{01}}{\gamma}-\frac{1}{\gamma} \frac{F_{0}^{\prime}}{F_{0}}=0 \\
q_{t}-\frac{\nu_{10}}{\gamma} \frac{F_{0}}{F_{1}} e^{-\gamma(p-q)}+\frac{\nu_{10}}{\gamma}-\frac{1}{\gamma} \frac{F_{1}^{\prime}}{F_{1}}=0 \\
p(S, T)=q(S, T)=h(S) .
\end{array}\right.
$$

By classical solutions of (15) we mean functions such that $p \in C((0,+\infty) \times$ $(0, T]) \cap C^{2,1}((0,+\infty) \times(0, T)), q \in C((0,+\infty) \times(0, T]), q_{t} \in C((0,+\infty) \times(0, T))$.

Note that

$$
\begin{aligned}
& \gamma p=\nu_{10}(T-t)+\ln F_{0}(t)+u(S, T-t) \\
& \gamma q=\nu_{10}(T-t)+\ln F_{1}(t)-\ln \left(\nu_{10} \int_{0}^{T-t} e^{-u(S, s)} d s+e^{-\gamma h(S)}\right),
\end{aligned}
$$


since $p(t)=\gamma^{-1}\left(r^{0}+\ln F_{0}(t)\right)$ and $q(t)=\gamma^{-1}\left(r^{1}+\ln F_{1}(t)\right)$. Then, a comparison principle in $(p, q)$ solutions will be equivalent to a comparison principle for the $\left(r^{0}, r^{1}\right)$ variables.

We consider growth conditions analogous to (4)

$$
|p|,|h| \leq A \exp \left(\alpha \ln ^{2} S\right)=A S^{\alpha \ln S},
$$

for some positive constants $A$ and $\alpha$.

Corollary 2.3. Let $\left(p_{1}, q_{1}\right)$ and $\left(p_{0}, q_{0}\right)$ be two classical solutions of the system (15) corresponding to terminal data $h \equiv h_{1}(S)$ and $h \equiv h_{0}(S)$, respectively. If there exist some positive constants $A$ and $\alpha$ such that $p_{i}(S, t)$ and $h_{i}(S), i=0,1$ satisfy the conditions (18), then

$$
\begin{aligned}
& \inf \left(h_{1}-h_{0}\right) \leq p_{1}(S, t)-p_{0}(S, t) \leq \sup \left(h_{1}-h_{0}\right), \\
& \inf \left(h_{1}-h_{0}\right) \leq q_{1}(S, t)-q_{0}(S, t) \leq \sup \left(h_{1}-h_{0}\right) .
\end{aligned}
$$

In particular, let $h(S)$ be bounded from below (or from above) by a constant, i.e. $h(S) \geq h_{*}$ (resp. $h(S) \leq h^{*}$ ) and $p(S, t), q(S, t)$, be a classical solutions of the terminal value problem (15) satisfying (18). Then

$$
p(S, t) \geq h_{*} \text { and } q(S, t) \geq h_{*}\left(\text { respectively } p(S, t) \leq h^{*} \text { and } q(S, t) \leq h^{*}\right),
$$

for any $S \in(0,+\infty)$ and any $t \in(0, T]$.

Proof. The inequalities (19) follow immediately from Theorem 2.1] and representation (16). In order to prove (20) we will use (17), i.e.

$$
q_{i}(\cdot, t)=\gamma^{-1}\left[\nu_{10}(T-t)+\ln F_{1}(t)-\ln \left(\nu_{10} \int_{0}^{T-t} e^{-u_{i}(\cdot, s)} d s+e^{-\gamma h_{i}(\cdot)}\right)\right],
$$

for $i=0,1$. Similarly to the proof of Lemma 2.2 we derive

$$
\begin{aligned}
q_{1}(\cdot, t)-q_{0}(\cdot, t)= & -\gamma^{-1} \int_{0}^{1} \frac{d}{d \xi}\left[\ln \left(\nu_{10} \int_{0}^{T-t} e^{-u_{\xi}(\cdot, s)} d s+e^{-\gamma h_{\xi}(\cdot)}\right)\right] d \xi \\
= & \gamma^{-1} \int_{0}^{1} \frac{\nu_{10} \int_{0}^{T-t} e^{-u_{\xi}(\cdot, s)}\left(u_{1}(\cdot, s)-u_{0}(\cdot, s)\right) d s}{\nu_{10} \int_{0}^{T-t} e^{-u_{\xi}(\cdot, s)} d s+e^{-\gamma h_{\xi}(\cdot)} d \xi} \\
& \quad+\left(h_{1}(\cdot)-h_{0}(\cdot)\right) \int_{0}^{1} \frac{e^{-\gamma h_{\xi}(\cdot)}}{\nu_{10} \int_{0}^{T-t} e^{-u_{\xi}(\cdot, s)} d s+e^{-\gamma h_{\xi}(\cdot)}} d \xi
\end{aligned}
$$

Now, (5) implies the estimates (20).

The second part follows immediately due to the fact that $p_{*}(S, t) \equiv h_{*}$ and $q_{*}(S, t) \equiv h_{*}$ are the solutions of the problem (15) with constant terminal condition $h \equiv h_{*}$. Indeed, if we formally substitute $p_{*}(S, t) \equiv h_{*}$ and $q_{*}(S, t) \equiv$ $h_{*}$ in (15), then we arrive at the conclusion that it is sufficient to check the following identities

$$
\begin{array}{r}
-\frac{\nu_{01}}{\gamma} \frac{F_{1}}{F_{0}}+\frac{d_{0}+\nu_{01}}{\gamma}-\frac{1}{\gamma} \frac{F_{0}^{\prime}}{F_{0}}=0, \\
-\frac{\nu_{10}}{\gamma} \frac{F_{0}}{F_{1}}+\frac{\nu_{10}}{\gamma}-\frac{1}{\gamma} \frac{F_{1}^{\prime}}{F_{1}}=0
\end{array}
$$


or equivalently

$$
\begin{aligned}
& F_{0}^{\prime}=-\nu_{01} F_{1}+\left(d_{0}+\nu_{01}\right) F_{0}, \\
& F_{1}^{\prime}=-\nu_{10} F_{0}+\nu_{10} F_{1},
\end{aligned}
$$

which follow directly from the definition of $F_{0}$ and $F_{1}$.

\section{$3 \quad$ Existence of weak solutions}

In this section we study the existence and uniqueness of weak solutions in suitable function spaces. First we introduce the weighted $L^{2}$ space

$$
L_{w}^{2}:=\left\{u:\|u\|_{0}^{2}:=\int_{0}^{+\infty} u^{2}(S) w(S) d S<\infty\right\},
$$

given a weight function $w>0$. Then we define a weighted Sobolev space as follows

$$
H_{w}^{1}:=\left\{u: u \in L_{w}^{2} \text { s.t. } S u^{\prime}(S) \in L_{w}^{2}\right\},
$$

with norm $\|\cdot\|_{1}$ such that $\|u\|_{1}^{2}=\|u\|_{0}^{2}+\left\|S u^{\prime}\right\|_{0}^{2}$.

Let $\xi:[0,+\infty) \rightarrow[0,1]$ be increasing, infinitely continuously differentiable function and such that $\xi \equiv 0$ on $[0,1 / 2]$ and $\xi \equiv 1$ on $[1,+\infty)$. We will use $\xi$ to construct a sequence $\left\{u_{\epsilon}\right\}$ of compactly supported functions converging in $H_{w}^{1}$ to a given element $u \in H_{w}^{1}$. More precisely, the following auxiliary result holds.

Lemma 3.1. Let $\xi_{\epsilon}(x):=\xi(x / \epsilon)[1-\xi(x \epsilon / 2)], 0<\epsilon<1$ and $u_{\epsilon}:=\xi_{\epsilon} u$. Then $u_{\epsilon} \rightarrow u$ in $H_{w}^{1}$, as $\epsilon \rightarrow 0$.

Proof. Note that $\left(u-u_{\epsilon}\right)^{\prime}=\left(1-\xi_{\epsilon}\right) u^{\prime}-\xi_{\epsilon}^{\prime} u$,

$$
S \xi_{\epsilon}^{\prime}(S)=(S / \epsilon) \xi^{\prime}(S / \epsilon)[1-\xi(S \epsilon / 2)]-(S \epsilon / 2) \xi^{\prime}(S \epsilon / 2) \xi(S / \epsilon)
$$

is uniformly bounded with respect to $\epsilon$ and $1-\xi_{\epsilon} \rightarrow 0$ as well as $S \xi_{\epsilon}^{\prime}(S) \rightarrow 0$ as $\epsilon \rightarrow 0$. Then the Lebesgue's dominated convergence theorem implies that $\left\|u-u_{\epsilon}\right\| \rightarrow 0$ as $\epsilon \rightarrow 0$.

Next, let $u(S)$ be twice continuously differentiable on $(0,+\infty)$ and denote the operator $\mathcal{L} u:=-\frac{1}{2} \sigma^{2} S^{2} u^{\prime \prime}$. Then after integration by parts we formally obtain:

$$
\begin{aligned}
(\mathcal{L} u, v)_{L_{w}^{2}} & =-\frac{1}{2} \sigma^{2} \int_{0}^{+\infty} w S^{2} u^{\prime \prime} v d S \\
& =\frac{1}{2} \sigma^{2} \int_{0}^{+\infty}\left[w S^{2} u^{\prime} v^{\prime}+\left(S \frac{w^{\prime}}{w}+2\right) w S u^{\prime} v\right] d S,
\end{aligned}
$$

provided that the integrals above are well-defined, $w$ is continuously differentiable and $w S^{2} u^{\prime} v \rightarrow$ as $S \rightarrow 0$ and $S \rightarrow \infty$. For example, the above holds when $v$ is continuously differentiable and with compact support.

Following the above observations we introduce the bilinear form:

$$
a(u, v):=\frac{1}{2} \sigma^{2} \int_{0}^{+\infty} w S u^{\prime}\left[S v^{\prime}+\left(S \frac{w^{\prime}}{w}+2\right) v\right] d S .
$$


If the weight function $w$ is twice continuously differentiable, and there exists a constant $C>0$, such that

$$
\left|S \frac{w^{\prime}(S)}{w(S)}\right|,\left|S^{2} \frac{w^{\prime \prime}(S)}{w(S)}\right| \leq C, \forall S \in(0,+\infty) .
$$

then the bilinear form $a(u, v)$ is continuous and semi-coercive on $H_{w}^{1}$, i.e.,

$$
\begin{aligned}
|a(u, v)| & \leq c\|u\|_{1}\|v\|_{1}, \quad \forall u, v \in H_{w}^{1} \\
a(u, u) & \geq \alpha\|u\|_{1}^{2}-\beta\|u\|_{0}^{2}, \quad \forall u \in H_{w}^{1}
\end{aligned}
$$

for some suitable constants $c>0, \alpha>0$ and $\beta>0$ which are independent of $u$ and $v$.

We can choose such weight function that the call option payoff function $h=\max \{S-K, 0\}$ belongs to the space $H_{w}^{1}$, for example, take $w:=(1+S)^{\gamma}$, where $\gamma<-3$.

In addition, we assume that

$$
\theta:=\int_{0}^{+\infty} w(S) d S<+\infty .
$$

This assumption guarantees that any bounded and measurable function belongs to $L_{w}^{2}$.

Lemma 3.2. There exists a constant $c_{0}>0$ such that

$$
|u(S)|^{2} \leq c_{0}\|u\|_{1}^{2} \frac{1}{S} \exp (C|\ln S|), \quad \forall u \in H_{w}^{1},
$$

where $C$ satisfies (26).

Proof. Note that there exists a constant $c_{0}$ such that

$$
|u(1)|^{2} \leq c_{0}\|u\|_{1}^{2}, \quad \forall u \in H_{w}^{1},
$$

due to the Sobolev embbeding theorem.

Let $S$ be fixed and denote $v(\zeta):=u(\zeta S)$. We have

$$
\begin{aligned}
\|v\|_{1}^{2} & =\int_{0}^{+\infty} w(\zeta)\left(\zeta^{2} S^{2}\left(u^{\prime}(\zeta S)\right)^{2}+u^{2}(\zeta S)\right) d \zeta \\
& =\int_{0}^{+\infty} \frac{w(\zeta)}{S w(\zeta S)} w(\zeta S)\left(\zeta^{2} S^{2}\left(u^{\prime}(\zeta S)\right)^{2}+u^{2}(\zeta S)\right) d(S \zeta) \\
& \leq \frac{1}{S} \exp (C|\ln S|)\|u\|_{1}^{2}
\end{aligned}
$$

since

$$
\frac{w(\zeta)}{S w(\zeta S)}=\frac{1}{S} \exp \left(\int_{\zeta S}^{\zeta} \frac{w^{\prime}(\xi)}{w(\xi)} d \xi\right) \leq \frac{1}{S} \exp (C|\ln S|) .
$$

Then (30) follows from (31) since $v(1)=u(S)$. 
The space $H_{w}^{1}$ is densely and continuously embbeded in $L_{w}^{2}$. We consider the Gelfand triples

$$
H_{w}^{1} \subset L_{w}^{2} \subset H_{w}^{*}
$$

and

$$
L^{2}\left(0, T ; H_{w}^{1}\right) \subset L^{2}\left(0, T ; L_{w}^{2}\right) \subset L^{2}\left(0, T ; H_{w}^{*}\right),
$$

where $H_{w}^{*}$ is the dual of $H_{w}^{1}$. Next, we define the set

$$
W(0, T):=\left\{u \in L^{2}\left(0, T ; H_{w}^{1}\right), \dot{u} \in L^{2}\left(0, T ; H_{w}^{*}\right)\right\},
$$

where $\dot{u}$ is the distributional derivative of $u$. It is well known (see Lions and Magenes [5]) that

$$
W(0, T) \subset C\left([0, T], L_{w}^{2}\right) .
$$

For simplicity we will further write $u(\tau)$ instead of $u(S, \tau)$ when this does not lead to misunderstanding. Recall that

$$
\mathcal{F}[\tau ; u, \gamma h]:=-\nu_{01} e^{u(\tau)}\left(\nu_{10} \int_{0}^{\tau} e^{-u(s)} d s+e^{-\gamma h}\right)+\kappa .
$$

Definition 3.3. A function $u \in W(0, T)$ is called weak supersolution (subsolution) of the initial value problem (11) if $u(0) \geq \gamma h$ (resp. $u(0) \leq \gamma h$ ) and for a.a. $\tau \in(0, T)$ the inequality

$$
\langle\dot{u}, v\rangle+a(u, v) \geq(\leq) \int_{0}^{+\infty} w \mathcal{F}[\tau ; u, \gamma h] v d S,
$$

holds for any nonegative $v \in H_{w}^{1}$. Respectively, the function $u \in W(0, T)$ is called weak solution of the initial value problem (1) if $u(0)=\gamma h$ and for a.a. $\tau \in(0, T)$ the equality

$$
\langle\dot{u}, v\rangle+a(u, v)=\int_{0}^{+\infty} w \mathcal{F}[\tau ; u, \gamma h] v d S, \quad \forall v \in H_{w}^{1},
$$

holds.

Next, we prove the following comparison principle for weak super/subsolutions satisfying growth conditions of type (41).

Theorem 3.4. Let $\bar{u}$ be a weak supersolution of the initial value problem (1) with initial data $h(S) \equiv \bar{h}$ and $\underline{u}$ be a weak subsolution corresponding to the initial data $h(S) \equiv \underline{h}$ where $\underline{h}$ and $\bar{h}$ are given and $\underline{h} \leq \bar{h}$. Assume in addition, that there exist positive constants $A$ and $\alpha$ such that

$$
|\underline{h}|,|\bar{h}|,|\underline{u}|,|\bar{u}| \leq A \exp \left(\alpha \ln ^{2} S\right)=A S^{\alpha \ln S},
$$

for a.a. $(S, t) \in(0,+\infty) \times[0, T]$.

Then $\underline{u} \leq \bar{u}$ for a.a. $(S, t) \in(0,+\infty) \times[0, T]$.

Denote $u:=\bar{u}-\underline{u}$. We will prove that $u_{-}:=\max \{-u, 0\}=0$ almost everywhere. Similarly to (9), we obtain that the following inequality holds for a.a. $\tau \in(0, T)$ and for any nonegative $v \in H_{w}^{1}$ with compact support in $(0,+\infty)$ :

$$
\begin{gathered}
\langle\dot{u}, v\rangle+a(u, v) \geq-\nu_{01} \nu_{10} \int_{0}^{\infty}\left(\int_{0}^{\tau} \delta(\tau, s)(u(S, \tau)-u(S, s)) d s\right) v(S) w d S \\
-\nu_{01} \int_{0}^{\infty}(u(S, \tau)-\tilde{h}(S)) v(S) \delta(\tau) w d S,
\end{gathered}
$$


where

$$
\delta(\tau, s):=\int_{0}^{1} e^{u_{\xi}(\tau)-u_{\xi}(s)} d \xi, \quad \delta(\tau):=\int_{0}^{1} e^{u_{\xi}(\tau)-\gamma h_{\xi}} d \xi,
$$

$u_{\xi}:=\xi \bar{u}+(1-\xi) \underline{u}, u(\cdot, 0) \geq \tilde{h}:=\gamma(\bar{h}-\underline{h}) \geq 0$ and $h_{\xi}:=\xi \bar{h}+(1-\xi) \underline{h}$.

It is sufficient to prove the following auxiliary result:

Lemma 3.5. Assume that $\tau_{1} \geq 0$ is such that for any $t \in\left[0, \tau_{1}\right]$ the inequality $\bar{u}(t)-\underline{u}(t) \geq 0$ holds a.e. on $(0,+\infty)$. Then the same inequality holds for any $t \in\left[0, \tau_{1}+\bar{\tau}\right]$, where $\bar{\tau}>0$ is a constant which depends only on $\alpha$ and $\sigma$.

Proof. Let $\omega$ be defined by (10) and $u_{\epsilon}:=u+\epsilon \omega$ where $u=\bar{u}-\underline{u}$. Then, assume that $\bar{\tau}$ is chosen as in the proof of Lemma 2.2 We will prove that $u_{\epsilon-}:=\max \left\{-u_{\epsilon}, 0\right\} \equiv 0$ for a.a. $(S, t) \in(0,+\infty) \times\left[\tau_{1}, \tau_{1}+\bar{\tau}\right]$. Note that there exist a closed interval $I_{\epsilon} \subset(0,+\infty)$ such that $u_{\epsilon-}=0$ on the set $\left((0,+\infty) \backslash I_{\epsilon}\right) \times$ $\left[\tau_{1}, \tau_{1}+\bar{\tau}\right]$ due to the conditions (38). Now, let $\varphi(S)$ be a smooth function with compact support in $(0,+\infty)$ such that $\varphi(S)=1$ on the interval $I_{\epsilon}$. Then $u_{\epsilon} \varphi \in L^{2}\left(\tau_{1}, \tau_{1}+\bar{\tau} ; H_{w}^{1}\right)$ and $\left(u_{\epsilon} \varphi\right)_{-}=u_{\epsilon-}$. Next, for any nonnegative $v \in H_{w}^{1}$ with compact support $\operatorname{supp} v \subset I_{\epsilon}$ we have $\varphi v=v, a(u \varphi, v)=a(u, v)$ and then

$$
\begin{aligned}
\left\langle\frac{d}{d \tau}\left(u_{\epsilon} \varphi\right), v\right\rangle+a\left(u_{\epsilon} \varphi, v\right)=\langle\dot{u}, \varphi v\rangle+\epsilon\langle\varphi \dot{\omega}, v\rangle+a(u \varphi, v)+\epsilon a(\omega \varphi, v) \\
=\langle\dot{u}, v\rangle+a(u, v)-\frac{1}{2} \epsilon \sigma^{2} \underbrace{\left(2 \omega^{\prime} \varphi^{\prime}+\omega \varphi^{\prime \prime}, v\right)_{L_{w}^{2}}}_{=0} \\
\geq-\nu_{01} \nu_{10} \int_{0}^{\infty}\left(\int_{0}^{\tau} \delta(\tau, s)(u(S, \tau)-u(S, s)) d s\right) v(S) w d S \\
\quad-\nu_{01} \int_{0}^{\infty}(u(S, \tau)-\tilde{h}(S)) v(S) \delta(\tau) w d S \\
\geq-\nu_{01} \nu_{10} \int_{0}^{\infty}\left(\int_{0}^{\tau_{1}} \delta(\tau, s) d s\right) u(S, \tau) v(S) w d S \\
\quad-\nu_{01} \nu_{10} \int_{0}^{\infty}\left(\int_{\tau_{1}}^{\tau} \delta(\tau, s)(u(S, \tau)-u(S, s)) d s\right) v(S) w d S \\
\quad-\nu_{01} \int_{0}^{\infty} u(S, \tau) v(S) \delta(\tau) w d S,
\end{aligned}
$$

i.e.,

$$
\begin{aligned}
& \left\langle\frac{d}{d \tau}\left(u_{\epsilon} \varphi\right), v\right\rangle+a\left(u_{\epsilon} \varphi, v\right) \geq-\nu_{01} \nu_{10} \int_{0}^{\infty}\left(\int_{0}^{\tau_{1}} \delta(\tau, s) d s\right) u_{\epsilon}(S, \tau) v(S) w d S \\
& \quad-\nu_{01} \nu_{10} \int_{0}^{\infty}\left(\int_{\tau_{1}}^{\tau} \delta(\tau, s)\left(u_{\epsilon}(S, \tau)-u_{\epsilon}(S, s)\right) d s\right) v(S) w d S \\
& \quad-\nu_{01} \int_{0}^{\infty} u_{\epsilon}(S, \tau) v(S) \delta(\tau) w d S,
\end{aligned}
$$

where we have used the fact that $u_{\epsilon}>u$ and $u_{\epsilon}(S, \tau)-u_{\epsilon}(S, s)>u(S, \tau)-$ $u(S, s)$ for any $s \in\left[\tau_{1}, \tau\right]$ since $\omega(S, \cdot)$ is increasing on that interval. Now, take $v=u_{\epsilon-}$ and note that $u_{\epsilon}=u_{\epsilon+}-u_{\epsilon-}, a\left(u_{\epsilon} \varphi, u_{\epsilon-}\right)=-a\left(u_{\epsilon-}, u_{\epsilon-}\right)$ and

$$
u_{\epsilon}(S, s) u_{\epsilon-}(S, \tau) \geq-u_{\epsilon-}(S, s) u_{\epsilon-}(S, \tau) \geq-\frac{1}{2}\left(u_{\epsilon-}^{2}(S, s)+u_{\epsilon-}^{2}(S, \tau)\right) .
$$


After integration with respect to $\tau$ form $\tau_{1}$ to $t \in\left[\tau_{1}, \tau_{1}+\bar{\tau}\right]$ the inequality (43) implies

$$
\frac{1}{2}\left\|u_{\epsilon-}(t)\right\|_{0}^{2}+a\left(u_{\epsilon-}, u_{\epsilon-}\right) \leq-\int_{\tau_{1}}^{t}\left(\int_{0}^{\infty} \Sigma(S, \tau) u_{\epsilon-}^{2}(S, \tau) w d S\right) d \tau,
$$

where

$$
\Sigma(S, \tau):=\nu_{01} \nu_{10}\left(\int_{0}^{\tau_{1}} \delta(\tau, s) d s+\frac{1}{2} \int_{\tau_{1}}^{\tau} \delta(\tau, s) d s-\frac{1}{2} \int_{\tau}^{t} \delta(s, \tau) d s\right)+\nu_{01} \delta(\tau) .
$$

$|\Sigma(S, \tau)|$ is bounded from above by a constant, say $C>0$, when $S \in I_{\epsilon}$ and due to the semi-coercivity of the bilinear form $a(\cdot, \cdot)$ (see (28) ) we obtain:

$$
\frac{1}{2}\left\|u_{\epsilon-}(t)\right\|_{0}^{2} \leq(C+\beta) \int_{\tau_{1}}^{t}\left\|u_{\epsilon-}(\tau)\right\|_{0}^{2} d \tau
$$

Hence the Gronwall inequality implies $\left\|u_{\epsilon-}(t)\right\|_{0}=0$ for any $t \in\left[\tau_{1}, \tau_{1}+\bar{\tau}\right]$ since $\left\|u_{\epsilon-}\left(\tau_{1}\right)\right\|_{0}=0$. Then $u+\epsilon \omega \geq 0$ a.e. Thus $u \geq 0$ a.e. since $\epsilon>0$ is arbitrary.

We further prove another useful estimate.

Lemma 3.6. There exists a constant $C>0$ such that

$$
\max _{t \in[0, T]}\|u(t)\|_{0}+\|u\|_{L^{2}\left(0, T, H_{w}^{1}\right)} \leq C\left(\|u(0)\|_{0}+\|\hat{u}\|_{W(0, T)}+\gamma\|h\|_{0}+1\right)
$$

for any weak subsolution $u$ and any function $\hat{u} \in W(0, T)$ satisfying $u \geq \hat{u}$.

Proof. Let $v \in H_{w}^{1}$ be some nonnegative function. We have

$$
\begin{aligned}
\langle\dot{u}, v\rangle+a(u, v) \leq & \int_{0}^{+\infty} w \mathcal{F}[\tau ; u, \gamma h] v d S \\
\leq & -\nu_{01} \nu_{10}\left(\int_{0}^{\tau}[u(\tau)-u(s)] d s, v\right)_{L_{w}^{2}} \\
& -\nu_{01}(u(\tau)-\gamma h, v)_{L_{w}^{2}} \\
& +\left(\kappa-\nu_{01} \nu_{10} \tau-\nu_{01}\right)(1, v)_{L_{w}^{2}} .
\end{aligned}
$$

Take $v=u-\hat{u}$ and integrate (47) with respect to $\tau$ from 0 to $t$.

$$
\begin{aligned}
\frac{1}{2}\|u(t)\|_{0}^{2}+a(u, u) \leq & \frac{1}{2}\|u(0)\|_{0}^{2}+\left.(u, \hat{u})_{L_{w}^{2}}\right|_{0} ^{t}+a(u, \hat{u})-\int_{0}^{t}\langle\dot{\hat{u}}, u\rangle d \tau \quad \text { (48) } \\
& -\nu_{01} \int_{0}^{t}\left(\nu_{10} \tau+1\right)\|u(\tau)\|_{0}^{2} d \tau+\nu_{01} \nu_{10} \frac{1}{2}\left\|\int_{0}^{t} u(\tau) d \tau\right\|_{0}^{2} \\
& +C_{1}\left(\|\hat{u}\|_{L^{2}\left(0, t, L_{w}^{2}\right)}+\gamma\|h\|_{0}+1\right)\|u\|_{L^{2}\left(0, t, L_{w}^{2}\right)} \\
& +C_{2}\left(\gamma\|h\|_{0}+1\right)\|\hat{u}\|_{L^{2}\left(0, t, L_{w}^{2}\right)} .
\end{aligned}
$$

Then a standard argument implies the estimate (46). 
Now, we prove the existence of weak solutions, provided that $h \in H_{w}^{1}$. The proof is based on the lower and upper solution method (cf. [7]). However, the exponential nonlinearity in (11) causes some very technical difficulties which have to be overcome.

Theorem 3.7. Assume that $h \in H_{w}^{1}$. Then there exist a weak solution $u$ to the initial value problem (11). Moreover, there exists a constant $C>0$ independent of $u$ such that

$$
\|\dot{u}\|_{L^{2}\left(0, T, L_{w}^{2}\right)}+\|u\|_{L^{\infty}\left(0, T, H_{w}^{1}\right)} \leq C\left(\|u(0)\|_{1}+1\right)
$$

Proof. We will present the proof in several steps.

Step 1. Let $h \in L_{w}^{2}$ be bounded. Then there exists a weak solution $u$ to the initial value problem (1). In addition, if $u(0)=\gamma h \in H_{w}^{1}$, then the inequality (49) holds with a constant $C$ independent of $u(0)$.

Note that we can conctruct appropriate couple of a supersolution $\bar{u}$ and a subsolution $\underline{u}$. Indeed, let the constant $c_{0}$ be such that $|\gamma h| \leq c_{0}$ and take $\underline{u}:=-c_{0}-\bar{M} t$ for some positive constant $M$. If $M$ is great enough then $\underline{u}$ is a subsolution. Analogously, $\bar{u}:=c_{0}+M t$ is a supersolution provided that $M \geq \kappa$. Next, according to (8) we can choose a constant $N>0$ such that

$$
N u(\tau)+\mathcal{F}[\tau ; u, \gamma h]=N u(\tau)-\nu_{01} e^{u(\tau)}\left(\nu_{10} \int_{0}^{\tau} e^{-u(s)} d s+e^{-\gamma h}\right)+\kappa
$$

is increasing in $u$, i.e.

$$
N u_{1}(\tau)+\mathcal{F}\left[\tau ; u_{1}, \gamma h\right] \geq N u_{0}(\tau)+\mathcal{F}\left[\tau ; u_{0}, \gamma h\right],
$$

for all $u_{0}$ and $u_{1}$ such that $\underline{u} \leq u_{0} \leq u_{1} \leq \bar{u}$. Now, we can construct a decreasing sequence of supersolutions $u_{0}:=\bar{u}, u_{1}, u_{2}, \ldots$ such that $u_{n+1}$ is the solution of the initial value problem

$$
\left\{\begin{array}{l}
\dot{u}_{n+1}-\frac{1}{2} \sigma^{2} S^{2} u_{n+1, S S}^{\prime \prime}+N u_{n+1}=N u_{n}+\mathcal{F}\left[\tau ; u_{n}, \gamma h\right] \\
u_{n+1}(S, 0)=\gamma h(S)
\end{array}\right.
$$

and $\underline{u} \leq u_{n} \leq \bar{u}$. A standard argument implies that $u_{n}$ converges to a weak solution of the problem (11). We omit the details.

Next, assume in addition that $h \in H_{w}^{1}$. Then $\dot{u} \in L^{2}\left(0, T ; L_{w}^{2}\right)$ and $u \in$ $L^{\infty}\left(0, T ; H_{w}^{1}\right)$ (see, e.g., Bonnans [1]) and the following parabolic estimate holds:

$$
\|\dot{u}\|_{L^{2}\left(0, T, L_{w}^{2}\right)}+\|u\|_{L^{\infty}\left(0, T, H_{w}^{1}\right)} \leq c_{0}\left(\|u(0)\|_{1}+\|\mathcal{F}[\cdot ; u, \gamma h]\|_{L^{2}\left(0, T, L_{w}^{2}\right)}\right)
$$

We will prove the stronger estimate (49). First, we have

$$
\begin{aligned}
-\frac{1}{2} \sigma^{2} S^{2} u_{S S}^{\prime \prime}= & \mathcal{F}[\tau ; u, \gamma h]-\dot{u} \in L^{2}\left(0, T, L_{w}^{2}\right) \\
-\frac{1}{2} \sigma^{2} \int_{0}^{t}\left(S^{2} u_{S S}^{\prime \prime}, \dot{u}\right)_{L_{w}^{2}} d \tau= & \frac{1}{2} \sigma^{2}\left(\frac{1}{2}\|u(t)\|_{1}^{2}-\frac{1}{2}\|u(0)\|_{1}^{2}\right) \\
& +\frac{1}{2} \sigma^{2} \int_{0}^{t}\left(S\left(S \frac{w^{\prime}}{w}+2\right) u_{S}^{\prime}-u, \dot{u}\right)_{L_{w}^{2}} d \tau
\end{aligned}
$$




$$
\begin{aligned}
\int_{0}^{t}(\mathcal{F}[\tau ; u, \gamma h], \dot{u})_{L_{w}^{2}} d \tau= & \int_{0}^{+\infty}\left(\int_{0}^{t} \frac{d}{d \tau}(\mathcal{F}[\tau ; u, \gamma h]) d \tau\right) w d S \\
& +\int_{0}^{+\infty}\left(\int_{0}^{t}\left(\kappa \dot{u}+\nu_{01} \nu_{10}\right) d \tau\right) w d S \\
\leq & |\kappa| \theta^{1 / 2} \int_{0}^{t}\|\dot{u}(\tau)\|_{0} d \tau+\nu_{01}\left(1+\nu_{10} t\right) \theta
\end{aligned}
$$

since

$$
\begin{aligned}
\frac{d}{d \tau}(\mathcal{F}[\tau ; u, \gamma h]) & =\frac{d}{d \tau}\left[-\nu_{01} e^{u(\tau)}\left(\nu_{10} \int_{0}^{\tau} e^{-u(s)} d s+e^{-\gamma h}\right)+\kappa\right] \\
& =-\nu_{01} e^{u(\tau)}\left(\nu_{10} \int_{0}^{\tau} e^{-u(s)} d s+e^{-\gamma h}\right) \dot{u}-\nu_{01} \nu_{10} \\
& =\mathcal{F}[\tau ; u, \gamma h] \dot{u}-\kappa \dot{u}-\nu_{01} \nu_{10}
\end{aligned}
$$

and

$$
\int_{0}^{t} \frac{d}{d \tau}(\mathcal{F}[\tau ; u, \gamma h]) d \tau=\mathcal{F}[t ; u, \gamma h]-\mathcal{F}[0 ; u, \gamma h] \leq \nu_{01}
$$

We multiply both sides of the equation $\dot{u}-1 / 2 \sigma^{2} S^{2} u_{S S}^{\prime \prime}=\mathcal{F}[\tau ; u, \gamma h]$ with $\dot{u}$ in $L_{w}^{2}$ and integrate from 0 to $T$. Then (51) and (53) imply

$$
\begin{aligned}
\int_{0}^{t}\|\dot{u}\|_{0}^{2} d \tau+\frac{1}{4} \sigma^{2}\|u(t)\|_{1}^{2} \leq- & \frac{1}{2} \sigma^{2} \int_{0}^{t}\left(S\left(S \frac{w^{\prime}}{w}+2\right) u_{S}^{\prime}-u, \dot{u}\right)_{L_{w}^{2}} d \tau \quad \\
& +|\kappa| \theta^{1 / 2} \int_{0}^{t}\|\dot{u}(\tau)\|_{0} d \tau+\frac{1}{4} \sigma^{2}\|u(0)\|_{1}^{2} \\
& +\nu_{01}\left(1+\nu_{10} t\right) \theta \\
\leq \tilde{C} & {\left[\int_{0}^{t}\left(\|u(\tau)\|_{1}+1\right)\|\dot{u}(\tau)\|_{0} d \tau+\|u(0)\|_{1}^{2}+1\right] }
\end{aligned}
$$

for some constant $\tilde{C}>0$. Now, a techical, but standard argument implies that (49) holds.

Step 2. Let $h \in H_{w}^{1}$ be bounded from below, i.e., $u(0)=\gamma h \geq c$. Then there exists a weak solution $u$ to the initial value problem (1). In addition, the inequality (49) holds.

Let $\xi_{\epsilon}(x)$ be defined as in Lemma 3.1, i.e., $\xi_{\epsilon}(x):=\xi(x / \epsilon)[1-\xi(x \epsilon / 2)]$. Step 1 implies that there exists a solution $u_{\epsilon}$ corresponding to the initial condition $u_{\epsilon}(0)=\xi_{\epsilon}(\gamma h-c)+c=\xi_{\epsilon} \gamma h+\left(1-\xi_{\epsilon}\right) c$ which is bounded. Moreover, $\xi_{\epsilon} \gamma h+(1-$ $\left.\xi_{\epsilon}\right) c \leq \gamma h$ increases as $\epsilon \downarrow 0$ and converges in $H_{w}^{1}$ to $\gamma h$. Then the comparison principle from Theorem 3.4 implies that the sequence $u_{\epsilon}$ is increasing as $\epsilon \downarrow$ 0 . Next, the estimate (49) and Lemma 3.2 imply that $u_{\epsilon}(S, \tau)$ converges to a finite limit $u(S, \tau)$ for any $(S, \tau) \in(0,+\infty) \times[0, T]$. What is more, $\dot{u}_{\epsilon}$ is weakly convergent to $\dot{u}(S, \tau)$ in $L^{2}\left(0, T ; L_{w}^{2}\right), u_{\epsilon}$ is weakly-* convergent to $u$ in $L^{\infty}\left(0, T, H_{w}^{1}\right)$ and $u$ satisfies the estimate (49). Then it is sufficient to prove that $\mathcal{F}\left[\tau ; u_{\epsilon}, \xi_{\epsilon} \gamma h+\left(1-\xi_{\epsilon}\right) c\right]$ is weakly convergent to $\mathcal{F}[\tau ; u, \gamma h]$ in $L^{2}\left(0, T ; H_{w}^{*}\right)$. First, note that

$$
\mathcal{F}\left[\tau ; u_{\epsilon}, \xi_{\epsilon} \gamma h+\left(1-\xi_{\epsilon}\right) c\right]=\dot{u}_{\epsilon}-\frac{1}{2} \sigma^{2} S^{2} u_{\epsilon, S S}^{\prime \prime}
$$


is bounded in $L^{2}\left(0, T ; H_{w}^{*}\right)$ and then there exists an element $\tilde{\mathcal{F}} \in L^{2}\left(0, T ; H_{w}^{*}\right)$ such that

$$
\mathcal{F}\left[\tau ; u_{\epsilon}, \xi_{\epsilon} \gamma h+\left(1-\xi_{\epsilon}\right) c\right] \underset{L^{2}\left(0, T ; H_{w}^{*}\right)}{\overrightarrow{\mathcal{F}} .}
$$

On the other hand, $\mathcal{F}\left[\tau ; u_{\epsilon}, \xi_{\epsilon} \gamma h+\left(1-\xi_{\epsilon}\right) c\right]$ is bounded from above by the constant function $\kappa$. Let $v \in L^{2}\left(0, T ; H_{w}^{1}\right)$ be some arbitrary nonnegative function. Then Fatou's lemma implies

$$
\begin{aligned}
\langle\kappa-\tilde{\mathcal{F}}, v\rangle & =\lim _{\epsilon \rightarrow 0}\left(\kappa-\mathcal{F}\left[\cdot ; u_{\epsilon}, \xi_{\epsilon} \gamma h+\left(1-\xi_{\epsilon}\right) c\right], v\right)_{L^{2}\left(0, T ; L_{w}^{2}\right)} \\
& \geq\langle\kappa-\mathcal{F}[\cdot ; u, \gamma h], v\rangle \geq 0,
\end{aligned}
$$

i.e.

$$
\mathcal{F}[\cdot ; u, \gamma h] \in L^{2}\left(0, T ; H_{w}^{*}\right) \text { and } \mathcal{F}[\cdot ; u, \gamma h] \geq \tilde{\mathcal{F}}
$$

Finally, we prove that in fact

$$
\mathcal{F}[\cdot ; u, \gamma h] \equiv \tilde{\mathcal{F}} \text {, i.e., }\langle\mathcal{F}[\cdot ; u, \gamma h], v\rangle=\langle\tilde{\mathcal{F}}, v\rangle \quad \forall v \in L^{2}\left(0, T ; H_{w}^{1}\right) .
$$

First, observe that, $v_{\epsilon}:=\xi_{\epsilon} v \rightarrow v$ as $\epsilon \rightarrow 0$ in $L^{2}\left(0, T ; H_{w}^{1}\right)$. Hence, it is sufficient to prove (59) for functions $v$ vanishing outside a set of the form $I \times[0, T]$ where $I \subset(0,+\infty)$ is some closed interval. According to estimate (49) and Lemma 3.2 (applied to the interval $I$ ) the functions $u_{\epsilon}$ and $u$ are uniformly bounded on $I \times[0, T]$. Then

$$
\langle\mathcal{F}[\cdot ; u, \gamma h], v\rangle=\lim _{\epsilon \rightarrow 0}\left(\mathcal{F}\left[\cdot ; u_{\epsilon}, \xi_{\epsilon} \gamma h+\left(1-\xi_{\epsilon}\right) c\right], v\right)_{L^{2}\left(0, T ; L_{w}^{2}\right)}=\langle\tilde{\mathcal{F}}, v\rangle .
$$

Step 3. Let $h \in H_{w}^{1}$. Then there exists a weak solution u to the initial value problem (10). In addition, the inequality (49) holds.

Consider a sequence of problems with initial condition

$$
u_{N}(S, 0)=\max \{\gamma h(S),-N\}, \quad N=1,2, \ldots
$$

Then the corresponding solutions $u_{N}$ form a decreasing sequence due to the comparison principle and Lemma3.2. Moreover, the pointwise $\operatorname{limit}_{N \rightarrow \infty} u_{N}(S, \tau)$ is finite for any $(S, \tau)$ since the inequality (49) holds for each function $u_{N}$. Then the proof follows similar arguments as in Step 2.

Finally, note that the uniqueness of the weak solution is a consequence of the comparison principle. More precisely, we have the following corollary.

Corollary 3.8. Assume that $h \in H_{w}^{1}$. Then there exists a unique weak solution $u \in W(0, T) \cap L^{\infty}\left(0, T, H_{w}^{1}\right)$ to the initial value problem (1). Moreover, the estimate (49) holds with a constant $C>0$ independent of $u$.

\section{Acknowledgement}

The research is supported by the European Union under Grant Agreement number 304617 (FP7 Marie Curie Action Project Multi-INT STRIKE - Novel Methods in Computational Finance). The second author is also supported by Bulgarian National Fund of Science under Project I02/20-2014. 


\section{References}

[1] J.F. Bonnans, Numerical analysis of partial differential equations arising in finance, Master 2 "Probabilités et Finance", Paris VI and Ecole Polytechnique: revision of Aug. 29, 2013, available at http://www.cmap.polytechnique.fr/ bonnans/notes/edpfin/edpfin.html

[2] R. Carmona (Ed.), Indifference Pricing: Theory and Applications, Princeton University Press, Princeton NJ, 2008.

[3] S.D. Hodges, A. Neuberger, Optimal Replication of Contingent Claims under Transaction Costs, Rev. Futures Markets 8, (1989), 222-239.

[4] T.S.T. Leung, A Markov-modulated stochastic control problem with optimal multiple stopping with application to finance, Decision and Control (CDC), 49th IEEE Conference, IEEE, (2010), 559-566.

[5] J.- L. Lions, E. Magenes, Problèmes aux Limites non Homogènes et Applications, Vols. I and II., Dunod, Paris, 1968.

[6] M. Ludkovski, Q. Shen, European option pricing with liquidity shocks, Int. J. Theor. Appl. Finan., 16, No. 7, Article ID 1350043, 30 p. (2013). ISSN 0219-0249

[7] C. V. Pao, Nonlinear parabolic and elliptic equations. Plenum Press, New York, 1992.

[8] T. Zhou, Indifference valuation of mortgage-backed securities in the presence of prepayment risk, Mathematical Finance 20 (2010), no. 3, 479-507. 